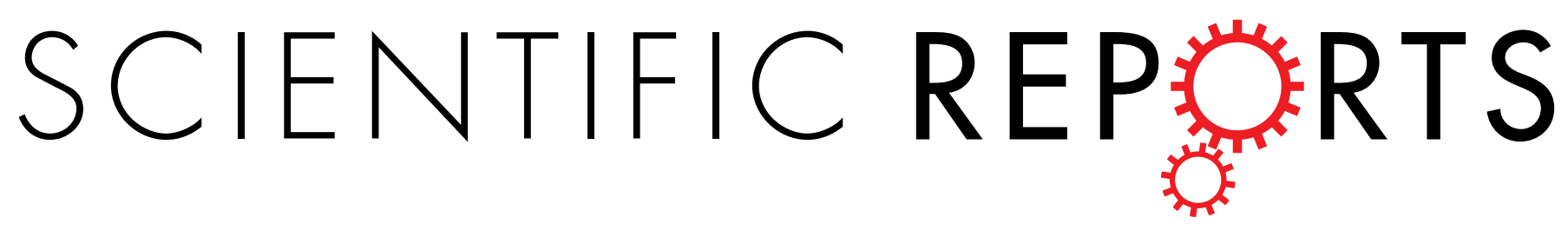

\title{
Corrigendum: Potent effects of dioscin against obesity in mice
}

Min Liu, Lina Xu, Lianhong Yin, Yan Qi, Youwei Xu, Xu Han, Yanyan Zhao, Huijun Sun, Jihong Yao, Yuan Lin, Kexin Liu \& Jinyong Peng

Scientific Reports 5:7973; doi: 10.1038/srep07973; published online 22 January 2015; updated 17 July 2015

This Article contains an error in Fig. 1c: the Sudan III staining for the 'ob/ob' group is incorrect. The correct Fig. 1C appears below as Fig. 1.

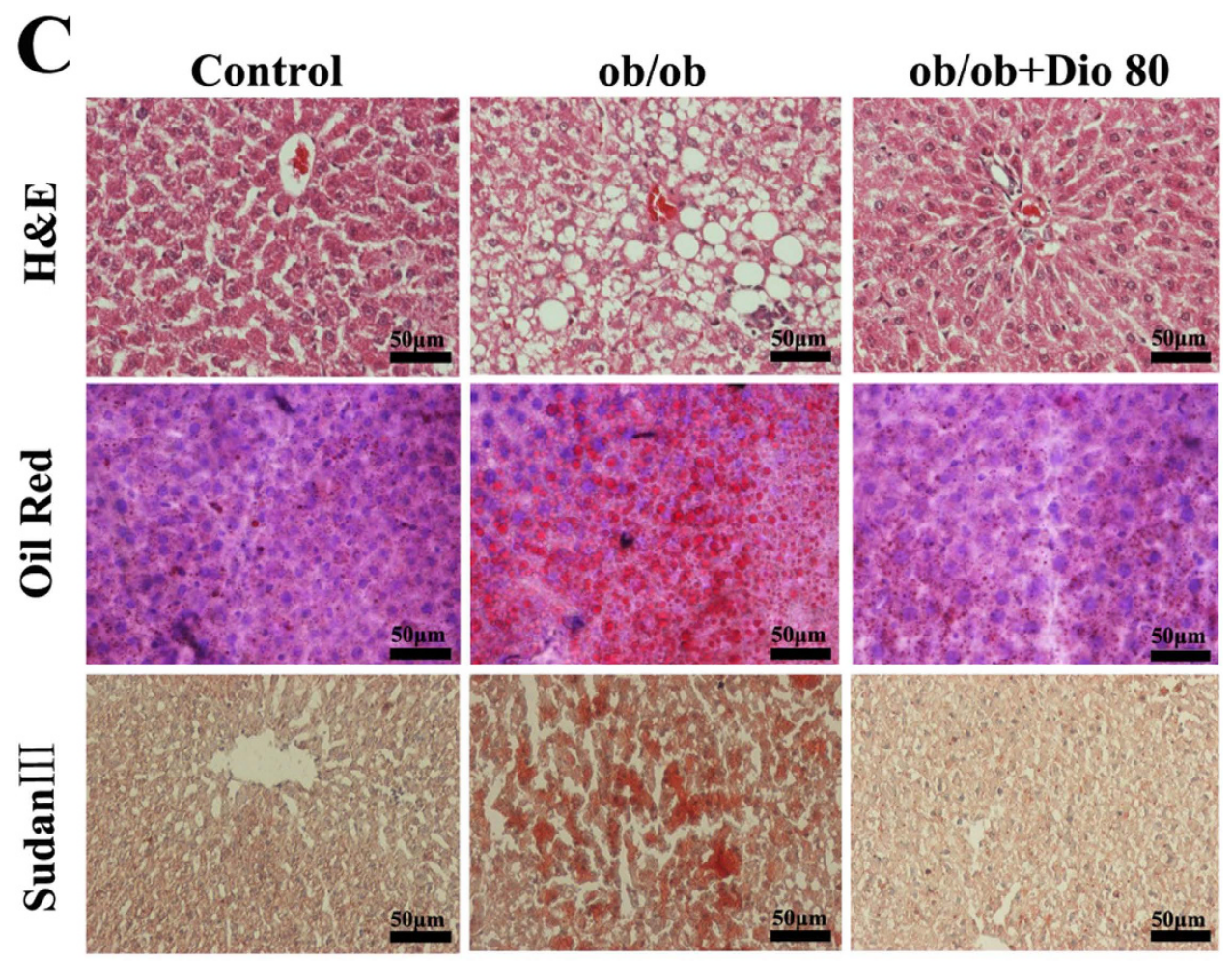

Figure 1. 